\title{
El síndrome de "echar la culpa" desde una mirada sistémica y sus repercusiones para la solución de problemas en la empresa*
}

\author{
Jorge Iván Pérez-Rave** \\ Favián González-Echavarría
}

Recibido: 14/12/2017 - Aceptado: 30/07/2018

https://doi.org/10.22395/rium.v17n33a10

\begin{abstract}
Resumen
Los objetivos del estudio son: explorar estructuras sistémicas del síndrome de "echar la culpa" en la empresa, describir metodológicamente una herramienta lúdica que ayuda a reconocerlo y mitigarlo, y discutir sus repercusiones para el análisis de causas y la solución de problemas. La exposición de la lúdica comprende descripción del caso, equipo de trabajo y ubicación, escenarios de observación (con y sin echar la culpa) y despliegue en tres grupos de prueba. Los resultados se comparan para ambos escenarios y se analizan causas de pedidos defectuosos, porcentaje de defectos y productividad. La lúdica puede ser empleada por docentes, consultores y formadores en general para enfrentar a los aprendices a un entorno simulado con el síndrome y sin este. Facilita reflexionar sistémicamente sobre el tema, identificar sus señales e intervenirlas antes de llevar a cabo el análisis de causas. Este trabajo aporta elementos teóricos y empíricos acerca de los beneficios de erradicar el síndrome de "echar la culpa" en análisis de causas, y propone considerarlo como factor contingente en futuros estudios.
\end{abstract}

Palabras clave: síndrome de echar la culpa; síndrome del sistema vulnerable; análisis de causas; aprendizaje lúdico; mejora continua.

* Artículo derivado del proyecto de investigación El síndrome de "echar la culpa" y su asocio con el éxito de los proyectos de mejora continua. En desarrollo desde abril de 2017 hasta julio de 2019. Financiación interna Idinnov SAS. Colombia.

** Director del grupo de investigación Idinnov. Medellín, Colombia. Correo electrónico: investigacion@idinnov. com. Orcid: https://orcid.org/0000-0003-1166-5545

*** Profesor de la Universidad de Antioquia, Faculad de Ingeniería, Departamento de Ingeniería Industrial. Medellín. Correo electrónico: favian.gonzalez@udea.edu.co. Orcid: https://orcid.org/0000-0002-1540-9859 


\title{
The "Blame-Blame" Syndrome from a Systemic Perspective and Its Implications for Problem Solving in a Company
}

\begin{abstract}
The objectives of the study are: to explore systemic structures of the "blame-blame" syndrome in a company, to describe methodologically a playful tool that helps to recognize and mitigate it, and to discuss its implications for causing analysis and problem solving. The ludic exposition includes case description, work team and location, observation scenarios (with and without blaming) and deployment in three test groups. Results are compared for both scenarios and causes of defective orders; percentage of defects and productivity are analyzed. Play can be used by teachers, consultants and trainers in general to confront learners in a simulated environment with and without the syndrome. It makes it easier to systemically reflect on the subject, to identify its signals and intervene before carrying out the cause analysis. This work provides theoretical and empirical elements about the benefits of eradicating the "blame-blame" syndrome in cause analysis, and proposes to consider it as a contingent factor in future studies.
\end{abstract}

Keywords: blame syndrome; vulnerable system syndrome; cause analysis; playful learning; continuous improvement.

\section{A síndrome de "colocar a culpa" a partir de uma perspectiva sistemática e suas repercussões para a solução de problemas na empresa}

\begin{abstract}
Resumo
Os objetivos do estudo são: explorar as estruturas sistêmicas da síndrome de "colocar a culpa" na empresa, descrever metodologicamente uma ferramenta lúdica que ajuda a reconhecê-la e mitigá-la, e discutir suas repercussões para a análise de causas e para a solução de problemas. A exposição da herramienta lúdica compreende a descrição do caso, da equipe de trabalho e da localidade, cenários de observação (com e sem colocar a culpa) e desdobramento em três grupos de teste. Os resultados são comparados para ambos os cenários e são analisadas as causas de pedidos defeituosos, porcentagem de defeitos e produtividade. A herramienta lúdica pode ser empregada por docentes, consultores e formadores em geral para enfrentar os aprendizes em um ambiente simulado com a síndrome ou sem ela. Ajuda a refletir sistemicamente sobre o tema, identificar seus sinais e intervir antes de realizar a análise de causas. Este trabalho fornece elementos teóricos e empíricos sobre os benefícios de erradicar a síndrome de "colocar a culpa" em análises de causas e propõe considerá-la como um fator contingente em futuros estudos.
\end{abstract}

Palavras-chave: síndrome de colocar a culpa; síndrome do sistema vulnerável; análise de causas; aprendizagem lúdica; melhoria contínua. 


\section{INTRODUCCIÓN}

El mejoramiento empresarial, desde la óptica del análisis-solución de problemas, implica avanzar desde una solución actual a otra deseada (de más generación de valor por parte de la organización), previa identificación e intervención de las causas que originan las brechas del caso, con alta participación de las personas y métodos sistemáticos (no necesariamente complejos). Así, la etapa de análisis de causas se constituye en un factor crítico para el progreso de las iniciativas de mejora, ya que termina guiando la planificación de las acciones, las futuras inversiones y las aspiraciones de los estrategas. A pesar de su amplio uso, los resultados no siempre son satisfactorios, ya que solo entre el $10 \%$ y el $30 \%$ de los proyectos de mejora tiende a lograr los resultados esperados [1], independiente del estilo de gestión en el que se enmarquen (Lean, Six Sigma, TPM, etc.).

Por años, los esfuerzos empresariales e investigativos se han centrado en técnicas y herramientas "duras" (o combinaciones entre ellas) para detectar y abordar las causas de los problemas y explicar el éxito de las iniciativas de mejora. A pesar de las múltiples metodologías propuestas y las configuraciones técnicas, los resultados no son concluyentes. Por ejemplo, Bessant et al. [2] en el año 1994 advertían sobre la necesidad de redescubrir la mejora continua y, más de veinte años después, McLean, Antony y Dahlgaard [1] coinciden con la mayor parte de los llamados del primero. Aun así, hoy día parece seguirse en la búsqueda de una receta universal que integre cinco porqués, diagrama causa efecto, gráficos Pareto, lluvia de ideas, entre otras herramientas.

No obstante, la comunidad académica ha comenzado a enfatizar sobre aspectos blandos de los equipos de trabajo y de su entorno, en favor del éxito de las iniciativas de mejora [3-4]. Al respecto, y esto se puede afirmar a partir de la teoría contingente (véase [5-6]), así la empresa cuente con conocimientos y habilidades técnicas y haga uso de una metodología fiable y válida, si el entorno no es apto para que todos estos elementos interactúen de una forma armónica, el desempeño se verá reducido.

En tal sentido, si la organización mantiene un entorno repleto del síndrome de "echar la culpa" (tendencia a culpar a las personas por resultados desfavorables, conocido como Vulnerable System Syndrome en áreas de la salud [7], o cercano al síndrome del "enemigo externo" en la teoría del pensamiento sistémico [8]), los análisis de causas derivarán en tensiones emocionales y respuestas "apaga incendios". Por ejemplo, las siguientes dos formas de explorar eventuales causas de un problema particular no necesariamente conducen por el mismo camino: 1) ¿Por qué dañó tantas piezas?, 2) ¿Qué condiciones pueden estar generando piezas defectuosas?

En el caso de la primera pregunta, el análisis de causas tenderá hacia situaciones propias del individuo, como: desconcentración, negligencia, incompetencia, indisciplina, etc. Este hace referencia a un modelo de análisis de causas centrado en la persona, 
el cual limita la responsabilidad y el control desde la organización [7-8]. En este caso, la aparente solución tiende a culminar con el despido del trabajador, por lo que no se propicia el aprendizaje del sistema.

La segunda pregunta se aparta de la persona, enfrenta las brechas como tensiones creativas y hace viable modificar el saber hacer del sistema. Las soluciones resultantes pueden mejorar procedimientos de entrenamiento, de auditoría de puntos clave, de selección, o de verificación de calidad; así como operaciones misionales, pruebas y ensayos, entre otros frentes. Es decir, evoluciona el saber hacer y se estimula el aprendizaje individual y colectivo.

La literatura ha venido alertando sobre la tendencia a echar la culpa en diversos ámbitos [9-12], y sobre lo negativa que resulta para el trabajo en equipo, el aprendizaje y la mejora de los procesos [13-15]. Sin embargo, este síndrome no ha sido lo suficientemente estudiado en contextos empresariales de análisis de causas y solución de problemas. De hecho, no se encontraron antecedentes de su operacionalización, ni tampoco ha tendido a tomarse en cuenta como constructo en los modelos estructurales en auge. Por el contrario, donde más se ha tratado ha sido en gestión de riesgos en áreas de la salud [7], por lo que el lenguaje, los síntomas, los patrones, las consecuencias y los tipos de errores, entre otros aspectos, no necesariamente son familiares o cercanos al análisis-solución de problemas en la empresa. De ahí que, además de ser pertinente tratar el síndrome de "echar la culpa" desde una óptica más comprensible para la gestión de empresas, también es pertinente proveer herramientas para detectarlo y hacerle frente. Más aún, cuando incluso en áreas de la salud se siguen reclamando trabajos que estimulen una cultura de detección y prevención de errores alejada del modelo prevaleciente, centrado en las personas [16].

Este artículo tiene como objetivo explorar la estructura sistémica del síndrome de "echar la culpa" en la empresa, describir metodológicamente una herramienta lúdica que ayuda a reconocerlo y mitigarlo, y discutir sobre sus repercusiones para el análisis de causas y la solución de problemas empresariales. El resto del documento se estructura así: la sección uno presenta varias razones, desde la literatura y desde un análisis sistémico, que sustentan por qué no echar la culpa en análisis-solución de problemas. La sección dos ofrece el desarrollo de la lúdica, comenzando por la descripción y finalizando por el despliegue en tres grupos de trabajo. La sección tres trata los resultados. La sección cuatro discute los hallazgos y propone desafíos. La sección cinco provee las conclusiones.

\section{ASPECTOS TEÓRICOS SOBRE ¿POR QUÉ NO ECHAR LA CULPA?}

Cuando se cae en la costumbre de echar la culpa durante el análisis-solución de problemas, la aparente causa raíz es la persona en la que recayó la manifestación del 
problema. Si esto es repetitivo, probablemente se opte por despedirla. Ahí termina el supuesto mejoramiento del sistema, en una variable dicotómica: con la persona, sin la persona, no más. El confort que deviene con el síndrome de "echar la culpa" es producido por el desconocimiento del mismo y por los círculos viciosos que este genera, algunos de los cuales se muestran en la figura 1 (véase más estructuras en [17]).

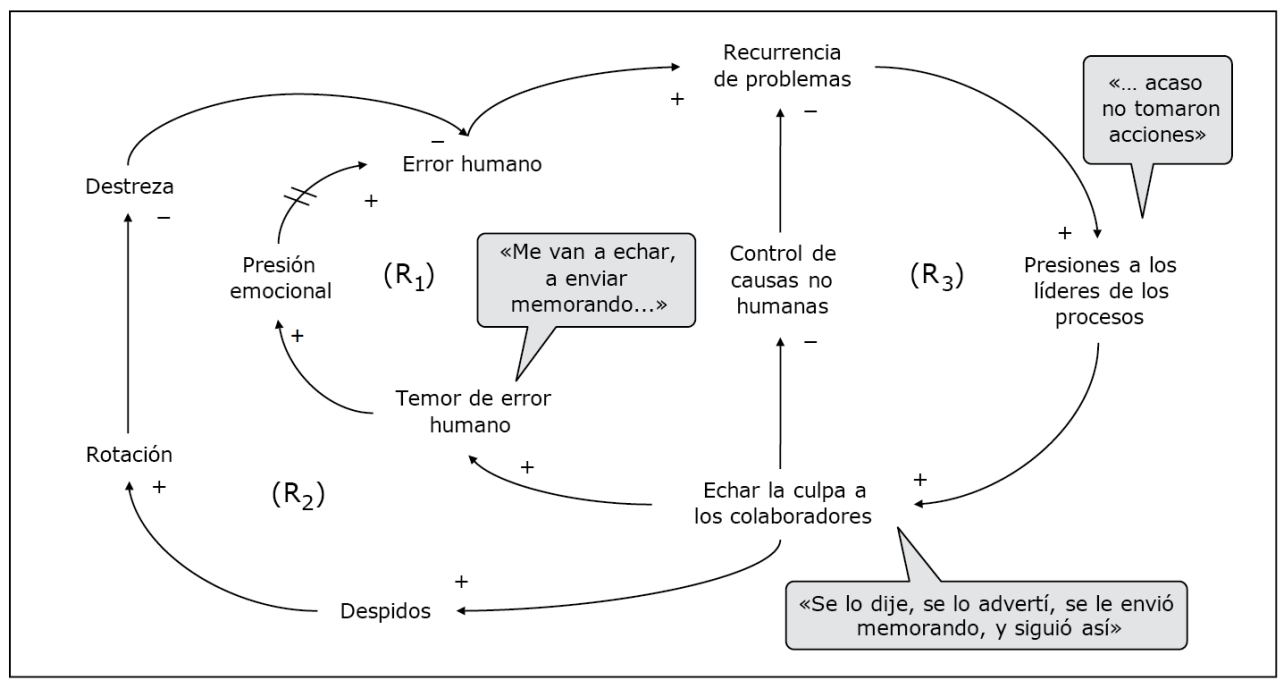

Figura 1. Algunos patrones subyacentes de "echar la culpa"

Fuente: [17]

Este diagrama (figura 1) se compone de varias estructuras sistémicas. Considere el origen de una determinada flecha como causa, y el fin de la flecha como efecto. La interpretación de estas relaciones se facilita a través de signos; el más $(+)$ deduce una relación directa (si uno aumenta, el otro también), y el signo menos (-) refiere una relación inversa (uno aumenta, el otro disminuye, y viceversa).

Para un mejor entendimiento de la figura 1, pártase del elemento Recurrencia de problemas. En la medida en que los problemas se repitan con mayor frecuencia, los afectados directamente (por ejemplo, clientes corporativos, en el caso de defectos), ejercerán presión sobre los responsables de los procesos (gerencia, calidad, producción, etc.), estos, a su vez, pueden verse tentados a seguir con el síndrome de "echar la culpa". Es decir, sus análisis de causas se inclinarán a culpar un determinado trabajador (ejemplo: quien estaba manipulando la máquina en la que se produjeron los pedidos defectuosos). Esta práctica impide explorar los múltiples factores no humanos, que es común agrupar en materiales, métodos, máquinas, mediciones y ambiente. Si estos no se identifican y controlan, sus posibles efectos no deseados seguirán impactando a lo largo del tiempo. 
Por otro lado, echar la culpa desencadena nuevas consecuencias. Por ejemplo, sensaciones de temor en los trabajadores, los cuales pueden estar exageradamente prevenidos a las fallas humanas. En el corto plazo, quizá se disminuyan los defectos causados por el factor humano. Sin embargo, esta aparente "solución" a un problema desencadena nuevos efectos (estrés, miedo, frustración, insatisfacción, ...). Estos no solo afectan la salud y contaminan el entorno, sino que además incrementan las posibilidades de error humano: justo lo que se quería evitar. Por lo mismo, aumenta la intensidad de los problemas, lo cual es consistente con [18], sobre el hecho de que el estrés, las altas cargas de trabajo y las crisis estimulan la generación de los errores.

Aquí no termina el desenlace, pues es probable que se le haya dicho en varias ocasiones al trabajador "ponga más cuidado", "esté más concentrado", etc. Aun así, ante la supuesta ineptitud del trabajador, la empresa opta por despedirlo. Con esto se fuga una parte del saber hacer de la organización y se deben ejecutar nuevos procesos de selección, contratación, entrenamiento, etc. Los trabajadores que ingresan, ante cuestiones de curva de aprendizaje, se convierten en fuente potencial de riesgos, como defectos, accidentalidad, ineficiencia, entre otros. Entonces, lo que aparentemente solucionó el problema (impacto en causas visibles) se constituye en el motivo de la recurrencia de problemas, debido, por un lado, a que no se toman en cuenta las otras fuentes de variación y, por el otro, a causa de los efectos latentes de echar la culpa (ejemplo: aspectos emocionales, ...).

En la anterior descripción son aplicables varias premisas del pensamiento sistémico, como: "la cura puede ser peor que la enfermedad", "soluciones rápidas que fallan" y "los problemas de hoy derivan de las soluciones del ayer" [8]. Sin embargo, el lector podría estar afirmando que, en el caso ilustrado, dichas premisas tienen validez en la medida en que las consecuencias de las demás causas ocultas (otros factores de variación), sean mayores que las del error humano. Pero, ¿qué tan válido resulta esto?

De acuerdo con gurús de la calidad como Joseph Juran, Philip Crosby y Edward Deming, es un error la tendencia a responsabilizar a los trabajadores por los defectos, pues como mínimo el $80 \%$ de las causas corresponden al sistema y están bajo el control de los estrategas (citado en: [17, 19, 20]). Según Ruiz, González y Alcalde se debe "evitar centrar la atención en culpar al individuo, pues, aunque se reconozcan los errores activos, lo que verdaderamente interesa es conocer los errores latentes del sistema para buscar posibles soluciones" [21]. Asimismo, Port manifiesta que las causas de errores del sistema ameritan soluciones del sistema; es decir, algo en el sistema permitió que el error se generara o no se detectara, por lo que no es culpa del trabajador [22]. 
Este síndrome también puede entenderse desde la teoría "X", la cual castiga el error y compensa el acierto [23]. Bajo esta óptica, en la medida en que el jefe obre bajo estos lineamientos se espera que los trabajadores desarrollen temor a los fallos, lo cual imposibilita el aprendizaje a través del error. Es decir, en lugar de considerar el error como una oportunidad de mejora, inherente al sistema, este se oculta y no da tiempo para reflexiones, análisis y soluciones, sino que prontamente se identifica un responsable. De esta manera, se desperdicia el potencial que tiene el intercambio recíproco de información para mejorar habilidades y cualidades de los individuos y, por ende, el desempeño de los procesos [24]. Entonces, el trabajador termina adoptando la misma postura "X" del jefe y evitará, a toda costa, la responsabilidad de un determinado comportamiento [25]. Además, dicho síndrome no solo puede surgir con la actividad laboral, sino desde los mismos hogares, la escuela, etc. Por ejemplo, si en la infancia o adolescencia el operador/gerente se vio atemorizado cada vez que se le relacionó con un fallo, recibiendo lo que se conoce como realimentación hacia el error, también puede presentar rasgos del citado síndrome.

En resumen, no solo desde factores internos sino también externos, la empresa es vulnerable a ser infectada con el síndrome "de echar la culpa". Por tanto, es evidente la necesidad de estrategias para reconocer y mitigar este síndrome en análisis - solución de problemas, con el fin de apartar la mirada de factores visibles e ir en busca de las causas latentes. Aquellas que pueden conducir a un mejoramiento verdadero y sostenido del sistema.

\section{DESARROLLO}

\subsection{Caso "Culpando al frutero"}

Esta lúdica fue creada por [17] y expuesta en formato de novela de ingeniería. En esta oportunidad, dicha herramienta se describe metodológicamente, con el fin de comprenderla desde el punto de vista técnico, estimular su reproducibilidad y nutrir la discusión sistémica sobre el síndrome en cuestión.

El caso "Culpando al frutero" [17] simula una empresa de venta de canastas de frutas. Recién ingresó a la empresa un nuevo frutero. Este ha acumulado tres meses en la compañía. A fin de mes se evaluará qué tan bueno habrá resultado para el trabajo asignado, que se describe en la figura 2.

Cuando acabe el turno de trabajo (luego de empacar 8 pedidos de los clientes), el inspector consolidará los resultados: pedidos defectuosos (no conformes), pedidos totales (8), duración efectiva del turno (se contabiliza el tiempo desde el primer pedido hasta el último). Con esta información, se calcula el porcentaje de generación de defectos (PGD) y la productividad (pedidos conformes/minuto de frutero). Cabe anotar que el 
número de frutas por pedido va aumentando, comenzando con tres y terminando con ocho frutas, lo cual es más difícil de memorizar.

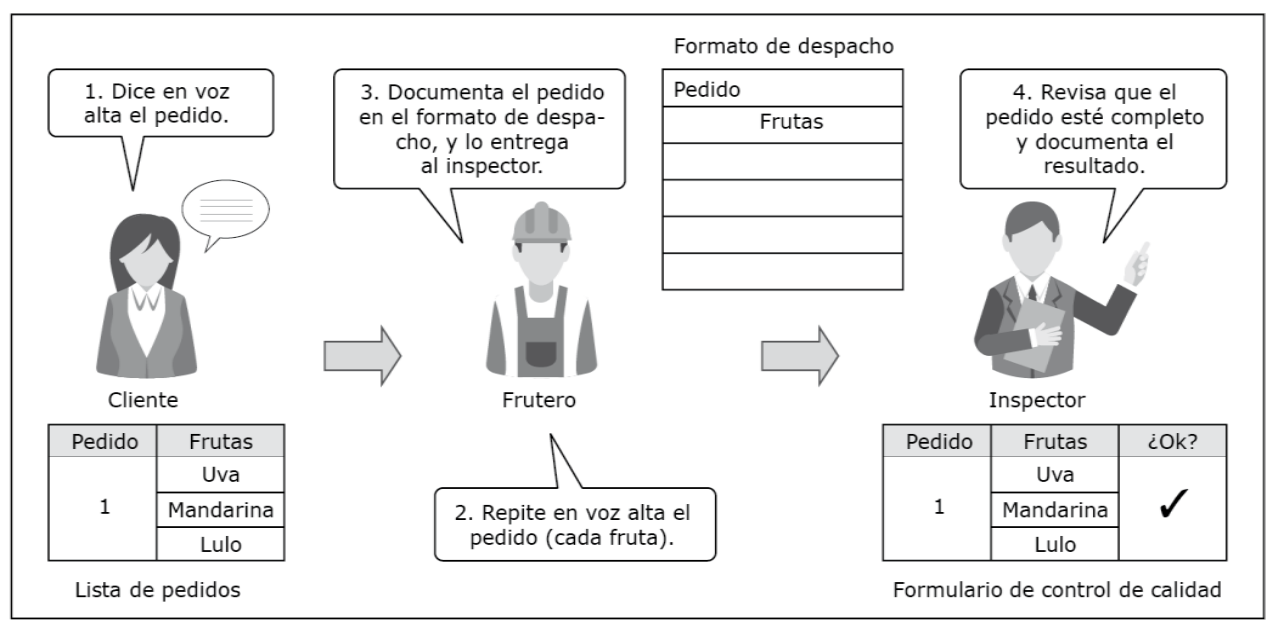

Figura 2. Procedimiento de producción de pedidos de frutas en escenario inicial.

$$
\text { Fuente: [17] }
$$

\subsection{Equipos de trabajo y ubicación}

En la lúdica intervienen tres personas: cliente, frutero(a) e inspector de calidad. Estas personas se ubican sentadas y cercanas entre sí, como se mostró en la figura 2. Es importante que tanto el inspector de calidad como el frutero tengan en sus asientos la posibilidad de apoyar sobre una base, puesto que les corresponderá escribir.

\subsection{Escenarios de observación y materiales}

Se simula dos escenarios de labor. El primero, llamado escenario con el síndrome de "echar la culpa", busca culpables durante la identificación de las posibles causas de pedidos defectuosos. En cambio, el segundo (sin presencia del síndrome) se enfoca en factores latentes que den lugar al aprendizaje del sistema.

Los materiales para el primer escenario son: dos lapiceros (uno para el frutero y otro para el inspector), un reporte de pedidos (cliente), un formato de despacho (frutero) $\mathrm{y}$ un formato de control de calidad (inspector). En [17] puede profundizarse al respecto.

\subsection{Despliegue}

El presente despliegue no busca generalizar conclusiones hacia una población específica. En vez de ello, pretende ilustrar la forma en que la citada herramienta lúdica 
posibilita crear un entorno simulado de trabajo para vivenciar síntomas y efectos del síndrome en cuestión, y propiciar la discusión de hallazgos. En ese sentido, "Culpando al Frutero" [17] se ejecutó en tres grupos de personas, con participación voluntaria, pertenecientes a una empresa manufacturera mediana. La conformación de cada grupo fue tal como se indicó en la figura 2 (cliente, frutero, inspector). Los grupos fueron intervenidos con la lúdica bajo los dos escenarios: 1) con síndrome de "echar la culpa" y 2) sin este. De cada escenario y grupo se tomaron dos observaciones, donde cada observación correspondió a ocho pedidos distintos. Así, se obtuvo un total de cuatro observaciones por grupo (o seis observaciones/escenario). En la figura 3, se resume el despliegue por grupo.

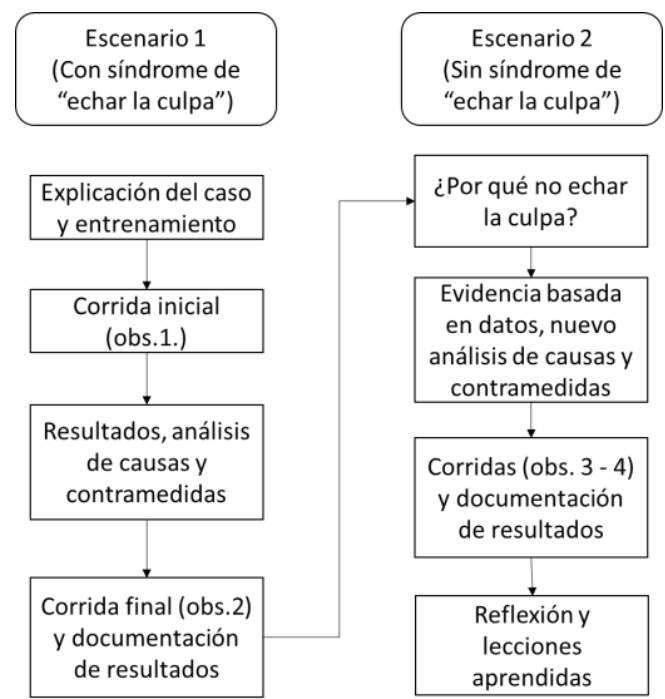

Figura 3. Secuencia de despliegue de la lúdica por cada grupo Fuente: elaboración propia

El escenario con síndrome de "echar la culpa" fue el primero en desplegarse en cada grupo. Una vez terminó la primera corrida de producción, se documentaron los resultados, a través de dos indicadores: PGD y productividad (pedidos conformes/ minuto). Luego, se dio inicio al análisis de causas, mediante una pregunta centrada en la persona: ¿Por qué el frutero dañó tantos pedidos? Allí, se recopilaron ideas de posibles causas de los pedidos defectuosos, y luego, cada equipo definió e implementó la contramedida. Se dio inicio a la segunda corrida de producción y se documentaron los nuevos resultados. Seguido, vino el segundo escenario (sin el síndrome de "echar la culpa"). En él, se compartieron razones (sistémicas y teóricas) expuestas en la sección de ¿Por qué no "echar la culpa"? Además, el análisis de causas, en lugar de centrarse en la persona, se enfocó sobre ¿qué factores pueden estar ocasionando pedidos defec- 
tuosos?, previa socialización de datos sobre tasas de éxito de fruteros típicos. Al final del ejercicio, se dio espacio para reflexiones y para compartir lecciones aprendidas.

\section{RESULTADOS}

\subsection{Escenario con síndrome de "echar la culpa"}

Las ideas de posibles razones de pedidos defectuosos, encontradas en el primer escenario, giraron en torno a: frutero desconcentrado, poco comprometido, no capacitado, desmotivado, distracción desde los demás compañeros, ruido en el ambiente, y dificultad para retener tantas frutas (se mencionó en uno de los grupos). Los tres grupos aportaron, en su mayoría, ideas centradas en causas humanas, por lo que la contramedida fue cambiar el frutero o expresarle que "ponga más cuidado". En la tabla 1 se presentan los resultados (PGD y productividad).

Tabla 1. Resultados para escenario con síndrome de "echar la culpa"

\begin{tabular}{|l|c|c|c|c|c|c|}
\hline \multicolumn{1}{|c|}{ Grupos } & \multicolumn{2}{c|}{$G 1$} & \multicolumn{2}{c|}{$G 2$} & \multicolumn{2}{c|}{ G3 } \\
\hline Corridas (observaciones) & 1 & 2 & 1 & 2 & 1 & 2 \\
\hline Total pedidos & 8 & 8 & 8 & 8 & 8 & 8 \\
\hline Pedidos defectuosos & 4 & 3 & 3 & 4 & 3 & 3 \\
\hline Duración de la corrida (min) & 4.0 & 3.86 & 4.01 & 3.7 & 3.98 & 4.1 \\
\hline PGD del grupo & \multicolumn{7}{|c|}{$44 \%$} & $44 \%$ & \multicolumn{2}{c|}{$38 \%$} \\
\hline PGD del escenario & \multicolumn{7}{|c|}{$42 \%$} & 1.17 & 1.24 \\
\hline Productividad del grupo & \multicolumn{7}{|c|}{1.15} & \multicolumn{7}{c|}{} \\
\hline Productividad del escenario & \multicolumn{7}{|c|}{1.18} \\
\hline
\end{tabular}

Fuente: elaboración propia

Para este escenario (tabla 1), impregnado de "echar la culpa", el PGD consolidado fue $42 \%$ (20/48), con una productividad de 1,18 pedidos conformes/minuto.

\subsection{Escenario sin el síndrome de "echar la culpa"}

El facilitador reorienta el análisis hacia causas latentes, alejadas de culpables. En ese sentido, se compartió información expuesta en [17] sobre la probabilidad de éxito (pedidos conformes) de tres fruteros, según la cantidad de frutas de los pedidos (figura 4).

Véase, en la figura 4, que los fruteros 1 y 2 acertaron todos los pedidos de máximo 4 frutas. Después de esta cantidad, fue notoria la tendencia a fallar. El frutero número 3 fue capaz de memorizar hasta 5 frutas. Ahora bien, haciendo énfasis en un entorno libre de "echar la culpa", el nuevo análisis de causas se presenta en la figura 5. 


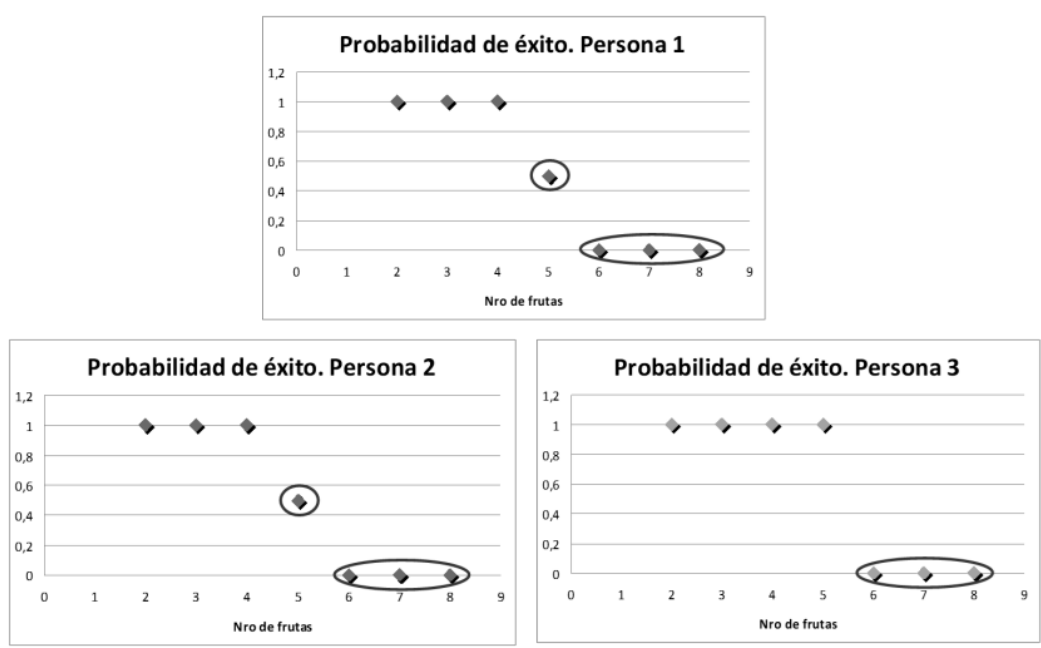

Figura 4. Información de apoyo en escenario sin el síndrome

Fuente: [17]

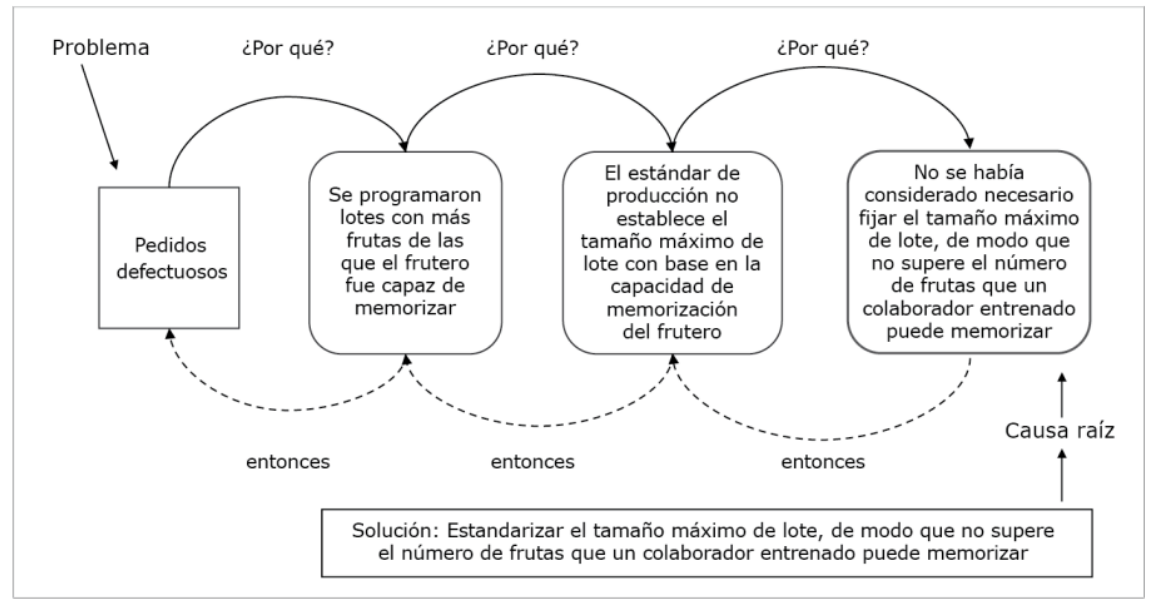

Figura 5. Diagrama de causas y consecuencias de "echar la culpa"

Fuente: [17]

Véase, en la figura 5, de izquierda a derecha, los cuestionamientos (¿por qué?) hasta llegar a la hipótesis de causa raíz del problema. Así mismo, de derecha a izquierda (parte inferior), nótese la coherencia entre causa - efecto, por medio del conector "entonces". Bajo esta óptica, como no se había considerado necesario fijar el tamaño máximo de lote con base en la cantidad de frutas que un frutero típico (entrenado y motivado) tiende a memorizar, entonces no existe tal estándar de producción. Por tanto, al no existir, al frutero se le hacen pedidos con más frutas de las que este suele recordar. En 
consecuencia, tiende a fallar en pedidos de 5 o más frutas. Nótese que la hipótesis es que la causa de los pedidos defectuosos se debe al diseño del estándar de producción y no a la persona. La solución propuesta es prácticamente la misma redacción que la causa, anteponiendo una acción; en este caso, estandarizar. En ese sentido, para intervenir dicha causa se dividió en dos partes (a y b) los pedidos con más de cuatro frutas, lo que lleva a estandarizar el tamaño máximo de lote, según la figura 6.

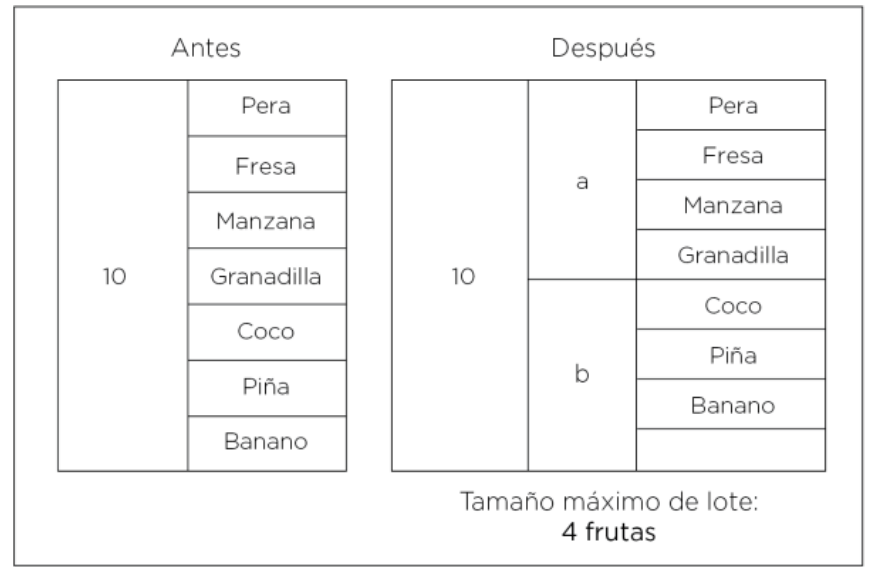

Figura 6. Nueva organización de los pedidos, ante el escenario sin síndrome de "echar la culpa"

Fuente: [17]

En este mismo escenario se aprovechó para mejorar el método de trabajo, atendiendo las ideas de mejora de los participantes. La figura 7 describe el nuevo método.

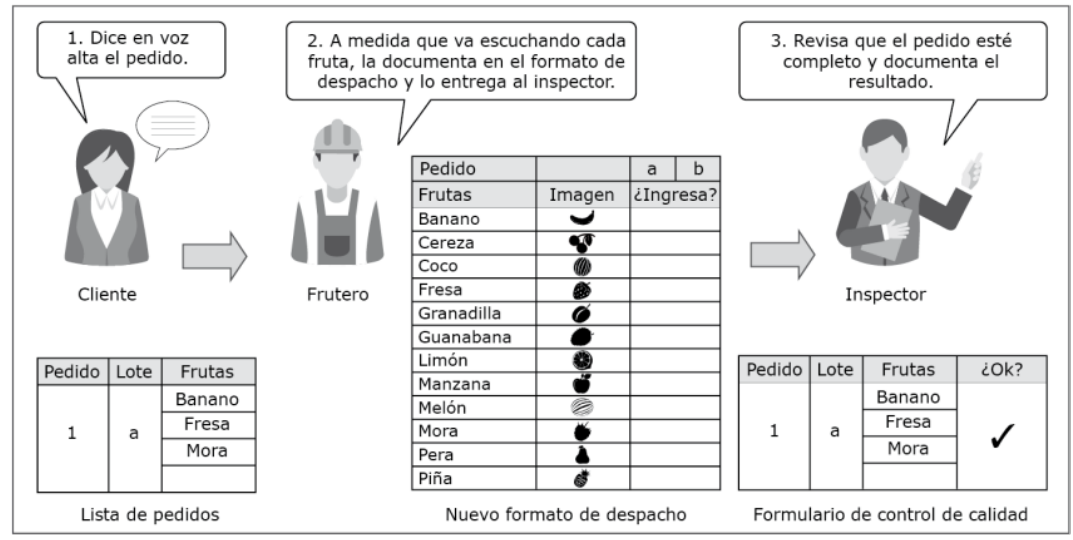

Figura 7. Procedimiento de producción de pedidos de frutas en escenario sin síndrome de "echar la culpa" 
Antes, el frutero debía repetir en voz alta el pedido y escribir cada fruta; pero, esta vez, el frutero solo debe marcar " $\checkmark$ " en la fruta que corresponda, a medida que el cliente hace el pedido. La tabla 2 ofrece los resultados obtenidos.

Tabla 2. Resultados para escenario sin síndrome de "echar la culpa"

\begin{tabular}{|c|c|c|c|c|c|c|}
\hline Grupos & \multicolumn{2}{|c|}{ G1 } & \multicolumn{2}{|c|}{ G2 } & \multicolumn{2}{|c|}{ G3 } \\
\hline Corridas (observaciones) & 3 & 4 & 3 & 4 & 3 & 4 \\
\hline Total pedidos & 8 & 8 & 8 & 8 & 8 & 8 \\
\hline Pedidos defectuosos & 1 & 0 & 0 & 0 & 0 & 0 \\
\hline Duración de la corrida (min) & 2.94 & 2.88 & 2.85 & 3.1 & 2.98 & 2.86 \\
\hline PGD del grupo & \multicolumn{2}{|c|}{$6 \%$} & \multicolumn{2}{|c|}{$0 \%$} & \multicolumn{2}{|c|}{$0 \%$} \\
\hline PGD del escenario & \multicolumn{6}{|c|}{$2 \%$} \\
\hline Productividad del grupo & \multicolumn{2}{|c|}{2.58} & \multicolumn{2}{|c|}{2.69} & \multicolumn{2}{|c|}{2.74} \\
\hline Productividad del escenario & \multicolumn{6}{|c|}{2.67} \\
\hline
\end{tabular}

Fuente: [17]

Para este escenario, vacunado contra el síndrome de "echar la culpa", el PGD consolidado fue solo del $2 \%$, con una productividad de 2,67 pedidos conformes/minuto.

\section{DISCUSIÓN}

Los resultados obtenidos bajo el escenario con el síndrome de "echar la culpa", en el entorno simulado de trabajo, son notablemente desfavorables, comparados con el escenario libre de búsqueda de culpables. En la figura 8 se muestran los resultados consolidados para ambos escenarios. Se destaca que, bajo el escenario libre del síndrome, el PGD se redujo en 95\% y la productividad aumentó en $126 \%$.
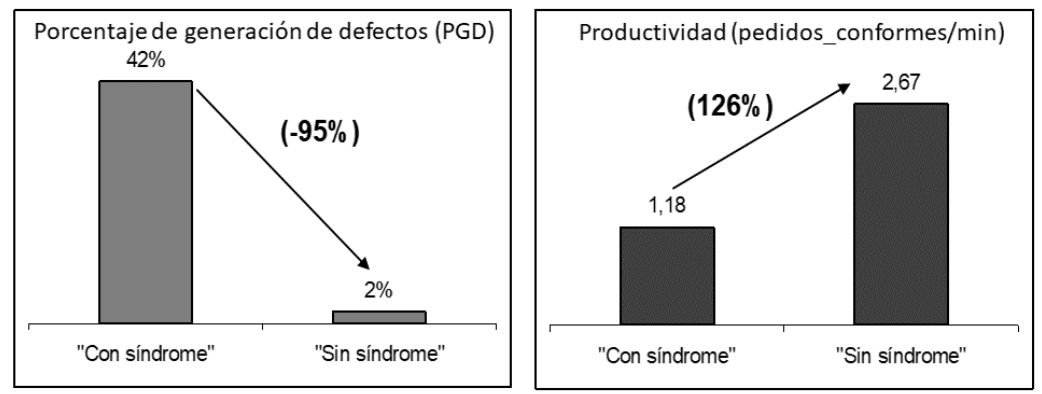

Figura 8. Comparación de resultados de ambos escenarios

Fuente: [17] 
Estos resultados lúdicos y el desenlace en general, motivan varias reflexiones en contextos de análisis - solución de problemas empresariales. Así, en un entorno impregnado de "echar la culpa", subyace un enfoque que considera al trabajador como un objeto de producción, al que hay que estar vigilando, supervisando para que "haga las cosas bien". Las soluciones ante este estilo de gestión son "se lo dije, se lo advertí, pero no hizo caso, no sirve". En cambio, bajo un escenario libre del síndrome de "echar la culpa", el trabajador es un aliado, con sumo conocimiento, que puede ayudar a encontrar e intervenir la causa raíz de los problemas. La gerencia confía en sus empleados, cuida de ellos y busca liberarles el potencial humano. Todo esto redunda, entre otras bondades, en el mejoramiento de la calidad de los productos y la reducción de los costos y gastos.

En el primer escenario, la pregunta que motivó el análisis de causas giró en torno a "por qué cometió el error". Esta forma de indagar tiende a estimular justificaciones en las personas, en lugar de la recopilación de los hechos. Mientras que, en el segundo escenario, que no indaga por causas humanas sino inherentes al sistema, se potencia la creatividad en busca de causas latentes que favorezcan el aprendizaje y permitan generar cambios en el saber hacer del sistema.

Otro aspecto por señalar, es la ausencia de información objetiva en el primer escenario. En tanto que el segundo estuvo acompañado de datos, procesados y resumidos en información estadística, que estimularon el proceso racional de toma de decisiones.

Es evidente que "echar la culpa" requiere menos recursos, tiempo e inversiones para fijar la contramedida, pero al final, los resultados hablan por sí solos. La práctica de "echar la culpa" conlleva a memorandos, llamados de atención y despidos, por lo que no hace más que tapar un hueco y destapar otros, cobrando validez premisas como "los problemas de hoy provienen de las soluciones del ayer" [8]. Bajo tal enfoque, los analistas de problemas evaden la responsabilidad de las decisiones que se toman o se dejan de tomar en el sistema, y se limitan, como en el caso de la lúdica, a concluir que los defectos se deben a "enemigos externos" (Ej: falta de concentración del frutero). Todo ello no hace más que deteriorar el sistema, pues genera un ambiente de tensión, de temor al despido, a descargos, a memorandos, etc. Incluso, puede ser tal la presión sobre la persona, que esta termina por convencerse de que es "culpable", lo cual puede reducir su autoeficacia y su autoestima. De este modo, la "solución" no es más que prometer que "pondrá más cuidado" y, con ello, los ciclos viciosos-latentes se presentan una y otra vez.

La tendencia a "echar la culpa" ha sido mencionada en diversos ámbitos. Por ejemplo, Argyris advierte que esta práctica es una reacción defensiva, incluso en 
personas de alta formación, y frustra la capacidad de aprender a aprender, lo que limita la mejora continua del individuo y de la organización [9]. Lamming, en contextos de cadenas de suministros, resalta la tendencia de buscar excusas y culpar a los demás por los problemas del sistema, sobre todo por parte de los directivos [10]. Kanji, en un escenario de gestión de la calidad en una empresa mediana, reporta la inclinación de jefes de departamentos a culpar a otros por la fabricación defectuosa [25]. Leape, refiriéndose a un escenario de instituciones de salud, señala "intensa presión sobre los individuos para ocultar los errores, negar la responsabilidad, distanciarse de ellos, culpar a los demás" [11, p.9]. Asimismo, [12], en una aplicación de la metodología Lean en el sector salud, se encontró, entre otros hallazgos, frustración e inclinación de diversos grupos profesionales por culpar a otros ante las dificultades. Adicionalmente, en [13] se resalta que cuando las personas son menos propensas a culpar a otros por los errores, se facilita el trabajo en equipo. Y, de hecho, se sabe que este último es un requisito indispensable para el éxito de las iniciativas de mejora. En esa vía, [14], en un contexto de satisfacción con el aprendizaje por parte de profesionales, señala, entre otras cosas, la importancia de no culpar a otros por las dificultades; que, en su lugar, es necesario responsabilizarse de los retos y afrontarlos con determinación en la búsqueda de la mejora continua. Sumado a ello, Yalçin, en un ambiente de enseñanzaaprendizaje del Lean bajo un nuevo método, recalca la necesidad de que los educadores derriben primero el hábito de a culpar a otros cuando los resultados académicos en los estudiantes no son satisfactorios [15]. En contextos de salud, en [7] se alerta que, al echar la culpa, la organización falla en aprender que los errores marcan el inicio de una investigación sobre el tema, en vez de constituirse en la conclusión de esta. Por lo que es de entender que tal síndrome sea referido como una de las patologías más nocivas para la organización [7].

A pesar de los constantes reportes de la presencia del síndrome de "echar la culpa" en diversos ámbitos y de sus consecuencias sistémicas, la literatura sobre mejoramiento empresarial (Lean, Six Sigma, TPM, y Gestión de la Calidad en general) no ha estudiado lo suficiente este tipo de manifestación. La mayoría de los estudios, aunque valiosos, giran en torno a proponer marcos de trabajo o metodologías para análisis - solución de problemas, entre ellos: [26-30]. Asimismo, los estudios de naturaleza teórica (Ej: $[1,2])$, si bien han contribuido con la comprensión de predictores del desempeño de las iniciativas de mejora, han tendido a considerar el factor macro "cultura/estilo". No obstante, dicho síndrome, a pesar de que está inmerso en tal factor, presenta particularidades que lo hace único; más aún, cuando tiene tanta influencia sobre una de las etapas más críticas de la solución de problemas: el análisis de causas.

Así, se generan varias oportunidades de estudio, entre ellas: seguir observando y retratando las manifestaciones sistémicas del citado síndrome en la empresa, 
crear una medida fiable y válida del constructo que representa, e incorporarlo como factor moderador/mediador, desde la Teoría Contingente, en modelos explicativos del desempeño. Esto resulta pertinente, teniendo presente el interés de la comunidad académica por explicar el desempeño de las iniciativas de mejora empresarial, a partir de constructos como: capacidad de absorción, liderazgo transformacional, cultura de aprendizaje, estrategia de innovación, etc. Todos ellos podrían verse favorecidos o reprimidos según la intensidad del síndrome de "echar la culpa" en la empresa.

Desde una óptica de análisis - solución de problemas en la empresa, este trabajo amplía y aporta patrones, explicaciones y hallazgos sobre cuatro aspectos clave acerca del síndrome en cuestión, reportados por [7] para contextos de salud ("vulnerable system syndrome").

El primer aspecto clave que [7] plantea es que, debido a tal síndrome, algunos sistemas son más susceptibles a los eventos adversos. Al respecto, el presente artículo ha aportado evidencia exploratoria proveniente de un entorno simulado de trabajo (razones centradas en la persona, PGD y productividad). Además, ha mostrado cómo, desde una mirada sistémica, la presencia de tal síndrome alimenta tensiones emocionales ("ponga más cuidado", "se lo dije/advertí", ...) en vez de tensiones creativas, lo cual conduce a la recurrencia de problemas. El segundo aspecto planteado por [7] es la perpetuación de los niveles superiores de culpar a los trabajadores de primera línea, la tendencia a negar los problemas del sistema y la justificación de tales decisiones en el logro de las metas establecidas. Con relación a ello, se han citado y explicado estructuras sistémicas concretas (ciclos viciosos), las cuales se han interpretado desde contextos empresariales de análisis - solución de problemas. El tercero tiene que ver con la importancia de reconocer la presencia de este síndrome en las organizaciones, por lo que este artículo ha descrito metodológicamente una herramienta lúdica creada por Pérez-Rave [17] para revelar y reflexionar sobre la presencia de dicho síndrome. Para ello, tomando en cuenta el enfoque constructivista y el aprendizaje lúdico, dicha herramienta facilita que gerentes, personal táctico y operativo, docentes, estudiantes, etc., vivan la experiencia de trabajo en dos organizaciones simuladas; una que tiene el síndrome de "echar la culpa" y otra que lo ha derribado. El cuarto aspecto planteado por [7] es la necesidad de no quedarse en soluciones sintomáticas basadas meramente en acción - reacción, sino trascenderlas por medio de modificaciones constantes en los supuestos bajo los cuales se emprenden las acciones. Para ello, este artículo ha aportado razones de gurús de la calidad, de otros trabajos de referencia y de exploraciones empíricas sobre porqué la mayoría de los errores en la empresa no corresponden a las personas sino al sistema. 


\section{CONCLUSIONES}

Este trabajo explora el síndrome de "echar la culpa" desde una mirada sistémica y aporta elementos para reflexionar sobre la necesidad de mitigarlo. Asimismo, describe la lúdica "Culpando al frutero" [17] desde una orientación metodológica, contraria a como fue expuesta originariamente en [17] (narrativa). Esta puede ser empleada por docentes, consultores y formadores en general para enfrentar a los aprendices a un entorno simulado de trabajo con el síndrome de "echar la culpa" y sin este. De esta manera, bajo un enfoque constructivista, facilita reflexionar sobre el tema, identificar sus señales e intervenirlas, antes de llevar a cabo el análisis de causas.

Desde un punto de vista teórico, se invita a profundizar en el síndrome de "echar la culpa" en las organizaciones, así como a incorporarlo, en futuras investigaciones explicativas, como posible factor moderador o mediador de relaciones entre constructos en auge. De hecho, según [7], en escenarios de salud, la habilidad de reconocer los síntomas de este síndrome es catalogada como un factor clave de éxito. Además, dicho autor recalca que no hay organizaciones libres de caer en manos de tal síndrome [7].

\section{REFERENCIAS}

[1] R. McLean, J. Antony y J. Dahlgaard, "Failure of Continuous Improvement initiatives in manufacturing environments: a systematic review of the evidence," Total Quality Management and Business Excellence, vol. 28, N.`3-4, pp. 219-237, 2017.

[2] J. Bessant, S. Caffyn, J. Gilbert, R. Harding y S. Webb. "Rediscovering continuous improvement," Technovation, vol. 14, N. ${ }^{\circ} 1$, pp. 17-29, 1994.

[3] R. Subramanian, "Soft-skills training and cultural sensitization of Indian BPO workers: A qualitative study," Communications of the IIMA, vol. 5, N.², pp. 11-24, 2015.

[4] A. Escrig-Tena, M. Segarra-Ciprés, B. García-Juan y I. Beltrán-Martín, “The impact of hard and soft quality management and proactive behaviour in determining innovation performance," International Journal of Production Economics, N. ${ }^{\circ}$ 200, pp. 1-14, 2018.

[5] L. Donaldson, The contingency theory of organizations, Londres: Sage Publications, 2001,

[6] J. Baron y D. Kreps, "Consistent human resource practices," California Management Review, vol. 41, N. ${ }^{\circ} 3$, pp. 29-53, 1999.

[7] J. Reason, J. Carthey y M. De Leval, "Diagnosing vulnerable system syndrome: an essential prerequisite to effective risk management," Quality in Healt Care, vol. 10, N. ${ }^{\circ}$ 2, pp. 21-25, 2001 .

[8] P. Senge, La quinta disciplina. El arte y la práctica de la organización abierta al aprendizaje, Ciudad de México: Naucalpan, 1998. 
[9] C. Argyris, "Teaching smart people how to learn," Harvard Business Review, vol. 69, N. 3, pp. 5-15, 1991.

[10] R. Lamming, "Squaring lean supply with supply chain management," International Journal of Operations \& Production Management, vol. 16, N. ${ }^{\circ}$, pp. 183-196, 1996.

[11] L. Leape, "Human factors meets health care: The ultimate challenge," Ergonomics in Design, vol. 12, N. ${ }^{\circ}$, pp. 6-12, 2004.

[12] S. New, M. et al., "Lean participative process improvement: outcomes and obstacles in trauma orthopaedics," PloS one, vol. 11, N. ${ }^{\circ}$, pp. 1-13, 2016.

[13] P. Castka, C. Bamber y J. Sharp, "Measuring teamwork culture: the use of a modified EFQM model," Journal of management development, vol. 22, N. ${ }^{\circ}$ 2, pp. 149-170, 2003.

[14] C. Mercado, E. Bayugo, Z. Leynes, C. Lontok, D. Medilla y J. Manongsong, "Accounting Students' Learning Satisfaction of Professional Subjects as Basis for Continuous Improvement," Asia Pacific Journal of Education, Arts and Sciences, vol. 3, N.¹, pp. 99-109, 2016.

[15] F. Yalçin, "A new method in education: Lean," Electronic Turkish Studies, vol. 12, N. 6 , pp. 811-826, 2017.

[16] I. Lynch, P. Roberts, J. Keebler, O. Guttman y P. Greilich Error, "Detection and Reporting in the Intensive Care Unit: Progress, Barriers, and Future Direction," Current Anesthesiology Reports, vol. 7, N.`3, pp. 310-319, 2017.

[17] J. Pérez-Rave. El legado de Robert: Novela de ingeniería para el mejoramiento empresarial, Medellín: Idinnov, 2015.

[18] J. Sexton, E. Thomas y R. Helmreich, "Error, stress, and teamwork in medicine and aviation: cross sectional surveys," Brit Med J., N. 320, pp. 745-749, 2000.

[19] D. Pérez-Arrieta. Modelo de análisis basado en el mejoramiento continuo para reducir la base de la pirámide de la seguridad: actos y condiciones inseguras y con esto el número de ocurrencias anormales de seguridad, medio ambiente y salud, Tesis de pregrado, Universidad de la Sabana, Bogotá, [En línea], acceso 05 de junio, 2017; Disponible: https://goo.gl/6i0ZPC

[20] M. Hernández. Proceso de investigación de accidentes laborales, estudio de resultados, verificación de la calidad de informes disponibles en la Asociación Chilena de Seguridad y definición de modelo de análisis de incidentes y procesos. Asociación Chilena de Seguridad ACHS, junio de 2010. [En línea], acceso 04 de junio, 2010; Disponible: http://www.fiso-web. org/Content/files/proyectos-premio-fiso/1170.doc

[21] P. Ruiz, C. González y J. Alcalde, "Análisis de causas raíz. Una herramienta útil para la prevención de errores," Revista de Calidad Asistencial, vol. 20, N.², pp. 71-79, 2005.

[22] J. Port, "Comprendiendo la variación. Análisis de causa para poner en práctica medidas correctivas," Quality Progress, [En línea], acceso 04 de junio, 2017; Disponible: http://asq. org/quality-progress/2012/03/back-to-basics/volviendo-a-los-fundamentos-comprendiendola-variaci.html 
[23] A. Vainikka y M. Young-Scholten, "Direct access to X'-theory: evidence from Korean and Turkish adults learning German," Language acquisition studies in generative grammar, vol. 31, N. 4 , 71-89, 1994.

[24] P. Whithaker, Cómo gestionar el cambio en contextos educativos, Madrid: Narcea, 2005.

[25] G. Kanji, "Implementation and pitfalls of total quality management," Total Quality Management, vol. 7, N. ${ }^{\circ}$, pp. 331-343, 1996.

[26] I. Alsyouf, U, Kumar, L. Al-Ashi y M. Al-Hammadi, "Improving baggage flow in the baggage handling system at a UAE-based airline using lean Six Sigma tools", Quality Engineering, vol. 30, N. ${ }^{\circ}$, pp, 432-452, 2018.

[27] L. Martin, K. Donohoe y D. Holdford, "Decision-Making and Problem-Solving Approaches in Pharmacy Education," American journal of pharmaceutical education, vol. 80, N. 3 , pp.1-6, 2016.

[28] K. Łyp-Wrońska, "World Class Manufacturing methodology as an example of problems solution in Quality Management System," Key Engineering Materials, vol. 682, pp. 342-349, 2016.

[29] C. Milner y B. Savage, "Modeling continuous improvement evolution in the service sector: A comparative case study," International Journal of Quality and Service Sciences, vol. 8, N.³, pp. 438-460, 2016.

[30] A. Camarillo, "Support to Continuous Improvement Process in Manufacturing Plants of Multinational Companies through Problem Solving Methods and Case-Based Reasoning Integrated within a Product Lifecycle Management Infrastructure," ICCBR, (Cuadernos de trabajo), pp. 259-261, 2015. 